\title{
La poétique de la Ville de Georges Rodenbach
}

\author{
The Poetics of the City in Georges Rodenbach's Works
}

\begin{abstract}
RÉSUMÉ
Georges Rodenbach est l'auteur d'ouvrages en prose et en vers explorant la résonance des signes reçus du monde extérieur sur la subjectivité individuelle. Ses fictions, poèmes et essais se penchent sur les interactions entre les personnages et la ville - généralement Bruges - dans laquelle ils évoluent, chargeant la réalité objective de symboles révélant leurs propres déterminations intimes. L'influence réelle ou symbolique de la Ville sur les développements narratifs, exprimée par une poétique de l'Analogie, est le sujet de cette étude, qui tentera de faire ressortir la cohérence globale des œuvres de Rodenbach à travers la permanence de sa « Poétique de la Ville».

Mots-clés : Georges Rodenbach, XIX ${ }^{\mathrm{e}}$ siècle, symbolisme, analogie, Bruges
\end{abstract}

\section{ABSTRACT}

Georges Rodenbach is the author of works in prose and verses exploring the resonance of the signs received from the outside world on the individual subjectivity. His fictions, poems and essays look into the interactions between the characters and the city - generally Bruges - in which they evolve, charging the objective reality with symbols revealing their own intimate determinations. The real or symbolic influence of the City on narrative developments, expressed by a poetics of Analogy, is the topic of this study, which will attempt to bring out the overall coherence of Rodenbach's works through the permanence of his "Poetics of the City".

Keywords: Georges Rodenbach, $19^{\text {th }}$ century, symbolism, analogy, Bruges

\section{Introduction. La ville extradiégétique à l'origine d'une poétique de la Ville}

L'œuvre du romancier, nouvelliste, poète et journaliste belge Georges Rodenbach (1855-1898), représentant parmi les plus caractéristiques du symbolisme fin-desiècle, s'organise autour de plusieurs topoï et récurrences, poétiques aussi bien que narratifs. Les interactions entre l'évolution intérieure des protagonistes et leurs déambulations dans l'espace d'une ville conçue soit comme leur interlocutrice privilégiée, soit comme leur alter ego, constituent l'un de ces principes unificateurs qui assurent la cohérence d'ensemble des écrits de l'auteur. De Bruges-la-Morte

David Paigneau, L'équipe de Recherche sur les Littératures, les Imaginaires et les Sociétés (ERLIS), Université de Caen-Normandie, Esplanade de la Paix, 14000 Caen, david.paigneau@lilo.org, https:// orcid.org/0000-0002-9561-5299 
au Carillonneur, en passant par les contes publiés dans Le Journal à partir de juin 1897, les articles et les poèmes, c'est la ville de Bruges qui, le plus souvent, endosse ce rôle de cadre, de double symbolique, voire de " commanditaire " des actions accomplies par les personnages. Le nom de Rodenbach est si bien associé à celui de la capitale flamande, que Camille Mauclair le désigna comme «l'historien moral et matériel de Bruges » dans la Revue des revues du 15 février 1899 (Mauclair, 1899, as cit. in Rime, 2016, p. 8). Par ailleurs, une légende tenace fait de Bruges son lieu de naissance, bien qu'il ait en réalité vu le jour à Tournai. Cependant, cette confusion est compréhensible, tant le destin de l'auteur semble lié à celui de la ville : c'est en effet à Bruges qu'il passa une grande partie de sa jeunesse, et c'est là que son grand-père, député, chirurgien et membre de la loge La Réunion des Amis du Nord, l'initia à la franc-maçonnerie. C'est également dans sa ville d'élection qu'il puisa une grande partie de l'inspiration dont ses vers puis sa prose furent nourris. Journaliste au Figaro après 1888, il publia pour le compte du journal une série d'articles, Agonies de villes, dont le premier fut naturellement consacré à Bruges.

Bien évidemment, nous ne devons pas nous cacher que la position du " poète brugeois exilé » ne fut pas uniquement une source d'inspiration, mais également la stratégie d'un écrivain désireux de se démarquer dans une scène littéraire parisienne déjà bien fournie. Rodenbach lui-même a fréquemment mis en avant cette appartenance régionale / nationale censée expliquer la singularité de sa poétique :

C'est après avoir délaissé notre Flandre natale, notre Flandre d'enfance et d'adolescence pour venir définitivement à Paris, que nous nous mîmes à écrire des vers et des proses qui en étaient le rappel [...] la Flandre que nous avons recréée et ressuscitée pour nous, dans le mensonge de l'art (Marx, 1994, p. 22).

Cependant, la lettre suivante, adressée à Émile Verhaeren depuis Gand, donne un éclairage plus précis sur les raisons de ce « délaissement» de Bruges et la nécessité $\mathrm{du}$ « mensonge de l'art» pour la « recréer» : « Notre peuple est avant tout positif et matériel. À la poésie par exemple, il n'entend pas un mot, et d'ailleurs l'esprit se rouille dans l'air bourgeois que nous respirons ici» (Maes, 1952, pp. 68-69).

Ce point de vue très baudelairien sur la détresse de l'homme d'imagination en Belgique, mis en regard de l'atmosphère propice à l'éveil du sentiment poétique que Rodenbach n'eut de cesse de prêter à Bruges, impliquait nécessairement que la présence de la Ville sous sa plume se manifestât essentiellement sous le prisme de l'absence et de la perte : thèmes perceptibles dès 1884 , dans le poème « Paysage de ville » (L'Hiver mondain) :

Le courant fait le bruit du satin que l'on froisse en venant se frotter aux arches du vieux pont.

Et les cloches, dans l'ombre où nul ne leur répond, 
ont tû [orthographe d'origine] leur chant d'airain aux clochers de paroisse.

Peu de passants et rien que la vague rumeur

d'un chariot lointain, là-bas, dans une rue ;

rien qu'une triste plainte incessamment décrue

comme le souffle lent d'une ville qui meurt! (Rodenbach, 1884, p. 67).

La « ville qui meurt», renvoi explicite à la chute de Bruges en tant que capitale commerciale $^{1}$, vient concrétiser un imaginaire poétique entièrement tourné vers le constat d'un néant qui ne fut pas toujours tel : le « chant d'airain » des cloches, auquel « nul ne répond », s'est « tu »; la « vague rumeur » du chariot est « lointaine »; la « triste plainte » est « incessamment décrue ». Selon cette grille de lecture, il n'est pas anodin que Françoise Chenet-Faugeras ait attribué à Rodenbach la paternité de l'expression « paysage urbain $»^{2}$ (Chenet-Faugeras, 1994, p. 27). Any BodsonThomas, quant à elle, avait décelé la signification profonde du titre Bruges-la-Morte dès lors qu'il était pris dans son sens littéral :

Hugues Viane représente, bien plus que le veuf éploré qui essaye en vain de retrouver dans une femme vivante le souvenir de celle qu'il a perdue, l'artiste amoureux de Bruges à qui la ville moderne n'offre qu'un très faible reflet de la ville ancienne, la seule aimée (Bodson-Thomas, 1942, p. 61).

Si l'esthétique de Georges Rodenbach peut, à bien des égards, être qualifiée de " poétique de la Ville », la biographie de l'auteur n'est donc pas étrangère à ce postulat. Les passerelles constamment dressées par le romancier et nouvelliste entre les mouvements passionnels de ses personnages et les « paysages urbains » qui les habitent autant qu'ils les habitent, prennent le plus souvent deux formes distinctes et complémentaires : celle d'une matérialisation de la mémoire et celle d'une détermination à agir. Ainsi, cette réflexion se propose d'analyser la " poétique de la Ville » rodenbachienne, non comme un topos stylistique commun à Bruges-la-Morte et au Carillonneur, mais comme un canevas symbolique central dans toute l'œuvre de l'écrivain, appuyant et illustrant des discours développés dans l'ensemble des genres littéraires qu'il a abordés. Ce canevas symbolique,

1 La baie du Zwin reliait Bruges à la mer du Nord, faisant d'elle l'un des plus importants ports commerciaux d'Europe jusqu'à son ensablement en 1475, qui coupa la ville de la mer, et par conséquent de la source de sa richesse, provoquant un déclin ininterrompu de plusieurs siècles. Rodenbach fait d'ailleurs directement référence à cet événement dans son article «Bruges » : «Un jour, en 1475, la mer du Nord brusquement se retira ; du coup le Zwyn fut tari, sans qu'on ait jamais pu le désensabler ou y rétablir une circulation d'eau; et Bruges, dorénavant éloignée de cette vaste mamelle de la mer qui avait nourri ses enfants, commença à s'anémier, et depuis quatre siècles elle agonise » https://journals.umcs.pl/1smll/ (1924, p. 15).

2 Dans l'« Avertissement » de Bruges-la-Morte (1892) : « [...] la Ville orientant une action; ses paysages urbains, non plus seulement comme des toiles de fond, comme des thèmes descriptifs un peu arbitrairement choisis, mais liés à l'événement même du livre » (Rodenbach, 1998, p. 50) [nous soulignons]. 
consistant à donner vie aux espaces urbains pour personnifier la mémoire et l'intériorité des protagonistes, se traduit, essentiellement, par la mise en place d'une « poétique de l'analogie ».

\section{2. "La ville rêve au beau passé qui finit mal » : mémoire et intériorité dans l'espace de la ville}

Dans les écrits de Rodenbach, la Ville apparaît le plus souvent affublée de la majuscule qui signale, avec une forme particulière de solennité voire de déférence, que l'espace urbain y représente beaucoup plus qu'un simple cadre géographique au sein duquel se déroulera l'action. En premier lieu, cette omniprésence est intimement liée à l'un des thèmes les plus structurants de la poétique rodenbachienne, celui de la nostalgie. Le veuf Hugues Viane a choisi de revenir vivre à Bruges parce qu'elle constitue le décor idéal d'une vie entièrement installée dans la déploration du bonheur passé : «Et comme Bruges aussi était triste en ces fins d'après-midi ! Il l'aimait ainsi ! C'est pour sa tristesse même qu'il l'avait choisie et était venu y vivre après le grand désastre " (Rodenbach, 1998, pp. 65-66). L'architecte Joris Borluut (Le Carillonneur) entend faire servir son art à une résurrection de la beauté perdue de la capitale flamande, à rebours du pragmatique Farazyn, tourné vers l'avenir et un projet de renaissance économique et commerciale. L'œuvre poétique de Rodenbach véhicule elle aussi l'obsession de l'auteur pour les motifs du temps, de l'absence et de la mémoire douloureuse, comme en témoigne « Douceur du souvenir» dans La jeunesse blanche :

\footnotetext{
Souvenir ! Ô douceur d'un amour qui s'achève!

Souvenir ! Ô douceur d'un songe qui n'est plus !

Rappel triste, en marchant, d'anciens vers qu'on a lus

Écume de la mer dont s'argente la grève (Rodenbach, 1978, p. 63).
}

Les motifs de la perte et de l'absence déterminent largement l'influence de la Ville sur les actions des protagonistes : la Bruges passée et «morte», ne trouvant son unique survivance que dans l'imaginaire prêté aux personnages, paraît d'autant plus les pousser à agir et dicter leurs choix qu'elle est en somme l'incarnation de leur subjectivité, impulsant de la sorte chaque étape des développements narratifs. L' ' Avertissement » de Bruges-la-Morte explicite ce rôle accordé par le romancier à la Ville-personnage :

Dans cette étude passionnelle, nous avons voulu aussi et principalement évoquer une Ville, la Ville comme un personnage essentiel, associé aux états d'âme, qui conseille, dissuade, détermine à agir (Rodenbach, 1998, p. 49).

Si la Ville est bien présentée ici comme « un personnage essentiel » dont l'impact sur l'arc narratif d'Hugues Viane sera primordial, c'est au sein d'une 
« étude passionnelle » explorant les associations entre Bruges et les « états d'âme », que cette influence s'exercera. Bien que les fictions de Rodenbach multiplient les emprunts au genre fantastique, l'auteur ne cache donc pas que le point de vue des protagonistes sur l'action exercée par la Ville exclut, dans la perspective du lecteur, toute interprétation réellement " surnaturelle » : le champ d'action du commanditaire symbolique ne dépasse jamais, dans les faits, les limites d'une « étude » centrée sur les turpitudes de l'âme humaine. Le conte Cortège, publié le 11 janvier 1898, illustre l'inséparabilité de l'intériorité - manifestée par la nostalgie - et de l'influence supposée de la Ville : Dorothée, espérant le retour de son amant dont elle conserve la bague comme symbole de son espoir, décide finalement de s'en séparer et d'en faire don aux autorités locales pour une souscription en vue de la reconstruction du beffroi. Ce geste mêle le destin du personnage à celui de la ville, puisqu'en sacrifiant l'ultime survivance matérielle de son amour perdu, Dorothée entérine son basculement définitif dans le territoire du passé tout en prenant part à la renaissance de l'espace urbain dans lequel elle évolue :

Instantanément, Dorothée comprit que c'était fini d'attendre. Le recommencement d'amour qu'elle espérait encore, et qui pouvait être, ne serait plus. C'est elle-même qui venait de se supprimer la dernière chance. La fiancé était mort pour elle. Elle avait créé l'irrémédiable. Et, comme la bague allait entrer dans la cloche, l'Amour de Dorothée entra dans le Souvenir. [...] Mais la bague avait consenti à mêler son peu d'or à tout le bronze anonyme du bourdon. Ainsi le chagrin d'amour de Dorothée, à cette minute, ne lui apparut plus distinct ni personnel, mais confondu avec l'universelle tristesse de la vie (Rodenbach dans Rime, 2016, pp. 180-182) [nous soulignons].

Gorceix note à ce sujet que le paradigme auquel se réfère le romancier, héritier tardif du romantisme allemand et inscrit dans les remises en cause des préjugés rationalistes au cours du XIX ${ }^{\mathrm{e}}$ siècle, tend en définitive à substituer les fantasmes du Moi à la description classique des œuvres réalistes : "Lorsque Rodenbach se propose de décrire 'l'ascendant' de Bruges sur les 'états d'âme' de son héros, il s'agit précisément de ces 'liens mystérieux et profonds' qui se tissent aux racines de l'être, dans le subconscient» (Gorceix, 1992, p. 18); « Le monde que Rodenbach présente, c'est celui qui est généré par la vision intérieure du personnage, le produit de son imagination » (Gorceix, 1992, pp. 19-20). L'influence de Bruges, au sein de ce canevas narratif, consiste donc à donner une apparence de justification à des actes finalement déjà inscrits dans la logique fictionnelle, ainsi que le confirme Aubert en prenant l'exemple du topos de l'eau dans les descriptions rodenbachiennes :

Or le paysage de Rodenbach présente un cas exemplaire de cette alliance nécessaire entre intérieur et extérieur [...], par l'utilisation d'un comparant majeur, l'eau, dont la signification allégorique et l'énorme rentabilité spéculaire permettent au poète de constituer le monde comme reflet autant que potentiel du rêve (Aubert, 2011, p. 102). 
Cette présence de l'eau brugeoise dans laquelle se reflète le destin des héros, n'est peut-être jamais aussi explicite que dans le conte La Ville (publié posthume le 15 juillet 1899) : deux amants s'installent à Bruges ${ }^{3}$ afin de pouvoir vivre plus intensément leur passion en se retirant dans un lieu éloigné de l'agitation du monde. Mais progressivement, l'atmosphère mortuaire de la ville dévitalise le sentiment amoureux ; la contemplation, par les protagonistes, de leurs reflets dans l'eau des canaux marque ce basculement d'Éros vers Thanatos :

Les journées s'écoulaient monotones. Mais est-ce que le vrai bonheur n'est pas monotone ? Ils allaient, au long des quais où songe une eau inanimée. Ils se regardaient parfois, du haut des ponts, dans cette eau des canaux. Eau vide, où il n'y avait qu'eux deux... Leurs visages étaient rapprochés l'un de l'autre, et se reflétaient, mais tout pâles, tout lointains, dans un recul pareil à celui de l'absence ou du souvenir. Mirés, ils apparaissaient si tristes ! On aurait dit qu'ils s'affligeaient de n'être déjà qu'un reflet, une image éphémère qui vacille et va sombrer jusqu'au fond... (Rodenbach, 2016, p. 144).

Les visages reflétés, semblant dans un premier temps personnifier l'idéal d'un bonheur amoureux au cœur duquel les amants croient être seuls au monde, dérivent rapidement vers une annonce de la séparation future, déjà inéluctable : le « recul » est « pareil à celui de l'absence ou du souvenir »; les personnages « s'affli[eai] ent de n'être déjà qu'un reflet » et une « image éphémère » dont le destin est de « sombrer jusqu'au fond ». Plus loin, c'est le son du carillon du Beffroi qui pousse le couple à interrompre ses ébats amoureux :

Les amants écoutaient, - inquiets d'on ne sait quoi. Est-ce que la ville religieuse en voulait à leur amour ? Et de trop vivre, en ces heures pâmées, provoqueraient-ils la mort dont c'est ici l'empire ? Hésitantes, leurs lèvres se reprenaient, après le carillon tu. Un long moment, les baisers gardaient un goût de cendre morte... Le carillon aussi leur fut comme le voisinage décourageant de la mort... (Rodenbach, 2016, p. 147).

Dans le dialogue final consommant la rupture, c'est donc sans surprise que les protagonistes attribuent à l'atmosphère néfaste de la Ville le dépérissement de leur amour : « Oui ! c'est la faute de la ville ! [...] Ce n'est pas de notre faute. La Mort ici fut plus forte que l'Amour » (Rodenbach, 2016, p. 150) Ainsi, les interactions entre espace extérieur et agitation intérieure transfigurent en une tragédie placée sous l'implacable tyrannie du Destin, ce qui pourrait n'être que la banale histoire d'un

3 Désignée par la périphrase " ville morte » : "Ils avaient choisi une ville morte, mise à la mode par des livres et des enthousiasmes de voyageurs, tout là-bas, au Nord, dans les brumes » (Rodenbach, 2016, p. 143) [nous soulignons]. Le jeune couple, au début du conte, incarne donc un « miroir inversé » d'Hugues Viane, lequel a choisi la « ville morte », non pour vivre plus intensément une passion amoureuse, mais pour se plonger plus profondément dans le deuil : "À l'épouse morte devait correspondre une ville morte. Son grand deuil exigeait un tel décor» (Rodenbach, 1998, p. 66) [nous soulignons]. 
amour ne survivant pas à l'extinction de la première passion. Dans la conclusion de Bruges-la-Morte également, Hugues Viane attribue à la volonté de la Ville un dénouement - le meurtre de Jane - inscrit en réalité dans la logique du déroulement narratif et - d'un point de vue intradiégétique - dans la destinée des protagonistes. Ainsi, Anny Bodson-Thomas notait, au sujet d'Hugues Viane : " Cet homme doux et sensible, miné intérieurement par la douleur qui lui a supprimé peu à peu sa volonté, s'est trouvé tout à coup sans défense devant un ordre du Destin » (BodsonThomas, 1942, p. 155). Toutefois, l'excipit laisse clairement entendre que, dans l'interprétation donnée par le personnage à son acte, la Ville approuve la mort de Jane Scott, condition indispensable pour retrouver le statut qui était le sien au début du roman - celui d'interlocutrice privilégiée du veuf éploré : «C'était fini, le beau cortège... tout ce qui avait été, avait chanté - semblant de vie, résurrection d'une matinée. Les rues étaient de nouveau vides. La ville allait recommencer à être seule» (Rodenbach, 1998, p. 273. Nous soulignons).

Enfin, notons que même lorsque la Ville retrouve un statut, plus classique, de cadre géographique, celui-ci se trouve intimement lié au sens profond de la fiction. Tel est par exemple le cas de Presque un conte de fées ${ }^{4}$, conte mettant en scène la Muse accompagnée de ses cygnes, chassés de partout jusqu'à ce que dans une ville - non spécifiée -, un poète lui fasse serment de l'aimer ainsi que ses compagnons :

Oui ! cette fois, on l'aimait, on l'aimait pour elle-même. Pur émoi du poète qui ne veut que les baisers de la Muse [...] Toujours il se trouve dans la ville un pur poète qui se met à m'aimer d'un amour assez pur et désintéressé pour que le prodige se renouvelle, pour que le linge soit changé en eau, et pour qu'ainsi mes cygnes ne meurent pas, que leur race dure et que la Poésie soit immortelle ! (Rodenbach, 2016, pp. 233-234).

Ainsi, bien que la ville dans ce conte ne soit pas explicitement Bruges, et bien qu'elle n'agisse pas sur les protagonistes à la manière de la Ville-Personnage, l'espace urbain y est néanmoins présenté comme celui où la Muse peut trouver un poète disposé à l'aimer - autrement dit : la Ville est par excellence le cadre propice à l'émergence d'une âme et d'une langue poétiques.

La présence de la Ville dans les écrits de Rodenbach personnifie donc avant tout les enjeux d'une plongée dans la subjectivité des acteurs des drames et la surimpression d'un " commanditaire symbolique » sur des retournements narratifs inévitablement inscrits dans la logique interne des récits. D'un point de vue stylistique, cette confusion volontaire entre les domaines de l'intériorité et de l'extériorité se manifeste le plus souvent par ce que nous pouvons nommer une « poétique de l'analogie », faisant porter sur des éléments variés de la fiction une signification dédoublée.

\footnotetext{
4 Publié posthume le 21 mars 1899.
} 


\section{Une poétique de l'analogie}

L'analogie est, au cœur des revendications à la fois esthétiques et métaphysiques portées par le courant symboliste, un procédé naturellement privilégié: conséquence logique d'une conception de la sensibilité comme outil de reconstruction du monde, déterminant l'écrivain à se voir comme un double réceptacle - de ses visions et de ses émotions. Le poète symboliste est celui qui doit réussir une synthèse entre extérieur et intérieur, observation et réinterprétation, pour placer le Moi du sujet perceptif au centre de ses préoccupations :

[L'évolution du symbole] consiste en un système sélectif de communication qui identifie, sans les clarifier, les domaines de la perplexité de l'homme, tels qu'ils apparaissent relativement au temps, aux lieux, et à la perception de la réalité d'un univers indifférent [...]. Le symbolisme établit un dialogue entre l'homme et son ombre ; il aspire non pas à une ascension vers une sphère supérieure, mais à une descente vers l'abîme (Balakia, 1997, pp. 376-388).

Il est donc parfaitement logique que l'analogie soit au cœur de la poétique rodenbachienne ; par ailleurs, l'auteur ne reculant pas devant les procédés didactiques intégrant une théorisation de sa propre esthétique jusqu'au sein des fictions, nous pouvons nous appuyer sur les justifications que lui-même nous a données de son utilisation des analogies. L' « Avertissement» de Bruges-la-Morte est bien sûr l'exemple le mieux connu de ces marques de didactisme ; toutefois, nous pouvons tout aussi bien mentionner l'incipit du conte Suggestion, publié posthume le 20 avril 1899 :

Il y a tout un domaine mystérieux et négligé, limbes de sensations, clair-obscur de la conscience, région équivoque où trempent pour ainsi dire les racines de l'être. Il s'y noue des analogies étranges, des rapports volatils qui lient nos pensées et nos actes à telles impressions de la vue, de l'ouïe, de l'odorat. Pour avoir rencontré une femme dont les yeux sont gris, l'homme du nord, tout à coup nostalgique, s'en retourne au pays natal. De même une orange qu'on épluche, parfois, suffit pour susciter toute l'atmosphère d'un théâtre. Et ceci encore : pour avoir respiré, sur un trottoir en réparation, l'été, l'odeur de l'asphalte qui bout dans sa cuve, nous partons pour la mer, avides de grands ports où le goudron sent bon aux quilles brunes des vaisseaux. Et ceci : les réverbères ophtalmiques, dans le brouillard, font rêver d'altruisme, de dévouements humanitaires, d'un legs pour un auspice ou une clinique des yeux... (Rodenbach, 2016, pp. 159-160).

Les allers-retours permanents entre extériorité (une femme aux yeux gris, une orange, l'odeur de l'asphalte...) et intériorité (la nostalgie du pays natal, le théâtre, la mer...) constituent un résumé assez exact de l'un des topoï les plus remarquables de l'écriture de Rodenbach. Notons en particulier l'effet de la femme aux yeux gris sur l'homme du Nord : l'analogie ici opérée rappelle notamment l'identification, dans Bruges-la-Morte, entre le gris de Ville et celui de l'âme du veuf :

De l'ensemble, c'est quand-même du gris qui émane, flotte, se propage au fil des murs alignés comme des quais. 
Le chant des cloches aussi s'imaginerait plutôt noir ; or, ouaté, fondu dans l'espace, il arrive en une rumeur également grise qui traîne, ricoche, ondule sur l'eau des canaux.

Et cette eau elle-même, malgré tant de reflets : coins de ciel bleu, tuiles des toits, neige des cygnes voguant, verdure des peupliers du bord, s'unifie en chemins de silence incolores.

Il y a là, par un miracle du climat, une pénétration réciproque, on ne sait quelle chimie de l'atmosphère qui neutralise les couleurs trop vives, les ramène à une unité de songe, à un amalgame de somnolence plutôt grise. [...]

Voilà pourquoi Hugues avait voulu se retirer là, pour sentir ses dernières énergies imperceptiblement et sûrement s'ensabler, s'enliser sous cette petite poussière d'éternité qui lui ferait aussi une âme grise, de la couleur de la ville ! (Rodenbach, 1998, pp. 130-131).

Par ailleurs, la couleur grise renvoie à un autre motif liant les caractéristiques de la Ville et la subjectivité des personnages, celui de la « cendre ». Rapprochement qui saute aux yeux en comparant ces deux extraits, respectivement tirés de l'article «Bruges » (Agonies de villes) et de Bruges-la-Morte:

Douceur de cheminer à présent dans la ville léthargique, à travers des songes et des souvenirs, au long des rues jamais droites, toujours capricieuses, ménageant, à chaque pas de la lente flânerie, une surprise et un imprévu [...]. Partout des ornements, un caprice, un symbole, un emblème, des armoiries ou des enseignes que le temps a patinés comme avec la cendre des années ! (Rodenbach, 1924, pp. 16-17) [nous soulignons].

Le veuf, ce jour-là, revécut plus douloureusement son passé, à cause de ces temps gris de novembre où les cloches, dirait-on, sèment dans l'air des poussières de sons, la cendre morte des années (Rodenbach, 1998, p. 54) [nous soulignons].

Par cette simple analogie récurrentes, Rodenbach illustre donc l'un des principes unificateurs centraux de son œuvre : l'interpénétration réciproque de l'atmosphère brugeoise et de l'évolution intérieure des protagonistes.

Le conte Déménagement, publié le 27 juin 1898, va encore plus loin dans cette systématisation des analogies entre intériorité et extériorité. Le narrateur se prépare à quitter son appartement tandis qu'une famille voisine s'apprête à enterrer sa jeune fille; tout l'argument du conte repose sur l'adéquation entre le sentiment de perte irrémédiable éprouvé par le personnage et le deuil - au sens propre - qui frappe ses voisins, et ce dès l'incipit :

Je n'oublierai jamais les impressions de ce dernier déménagement. Ceux qui changent souvent d'habitation s'aguerrissent, n'éprouvent pas cette douleur d'arrachement et d'adieu. Ils n'ont pas le temps de s'attacher aux lieux. Moi, je vivais là depuis dix années. Tout un morceau de ma vie qui, semblait-il, allait disparaître et s'y engloutir comme dans l'Éternité. Que de souvenirs suspendus en guirlandes fanées sur ces murs! Que d'illusions de jeunesse dédorées au fur et à mesure, en même temps que les dorures des salons, maintenant ternis ! Et les visages qui se mirèrent dans ces miroirs, aujourd'hui morts ou absents, et que j'allais voir émerger une dernière

5 Rappelons également que dans le conte La Ville, c'est d'« un goût de cendre morte » (Rodenbach, 2016, p. 147) [nous soulignons] que se gâtent les baisers des deux amants après que le son du carillon a interrompu leurs ébats. 
fois, comme si pour moi, ils n'existaient plus que là !

C'était en pleine chaleur de juillet. Je me trouvais un peu souffrant, au surplus, et prédisposé à m'émouvoir comme une sensitive. Ce déménagement me fut comme une petite mort, comme une répétition d'enterrement (Rodenbach, 2016, pp. 67-68).

L'analogie se double ici d'une prolepse, puisque la « répétition d'enterrement » annonce déjà les préparatifs qui s'effectuent, au même instant, dans l'appartement d'en face. De même, lorsque le narrateur trie les documents et manuscrits qu'il a choisi d'emmener avec lui et constate que la totalité tient dans une petite malle, l'analogie / prolepse se fait encore plus explicite : « Et cela ne pesa pas davantage qu'un cadavre d'enfant $\gg$ (Rodenbach, 2016, p. 70. Nous soulignons). La conclusion du conte, en clôturant les deux arcs narratifs parallèles, apporte dans le même temps une ultime touche à l'analogie :

Bientôt tout fut achevé. Mon appartement était vide. Je ne le reconnus guère... Plus rien de moi n'y était. Tout de suite, il fut lui-même. En bas, le vestibule de la maison, également, fut débarrassé avec promptitude.

Il ne garda pas plus longtemps trace de ma vie que l'autre n'avait gardé trace de la mort.

Et quand la voiture de déménagement s'achemina, tourna le coin de la rue, eut disparu, ce fut comme si un corbillard emportait la période vécue là, cette période de dix années (l'âge de la première communiante d'en face) qui était morte aussi ! (Rodenbach, 2016, pp. 74-75) [nous soulignons].

\section{Conclusion}

Nous pouvons conclure cette réflexion en constatant que la présence de la Ville sous la plume de Georges Rodenbach, dépasse de très loin le cadre d'un artifice poétique récurrent ou d'une personnification commune aux deux œuvres phares de l'auteur (commanditaire symbolique dans Bruges-la-Morte; Muse des idéaux de Borluut dans Le Carillonneur) : romans, articles, poèmes et contes reposent plus souvent qu'à leur tour sur l'étude des interactions entre les différentes nuances de tourments qui agitent l'âme humaine et la fixation, par la subjectivité du sujet perceptif, desdits tourments sur divers éléments de l'espace physique qui l'entoure. L'analogie, en tant que procédé chargé de donner une signification double, à la fois « intérieure » et « extérieure », aux objets décrits, constitue dans ce cadre l'une des marques les plus distinctives de la poétique rodenbachienne.

\section{References}

Aubert, N. (2011). Poésie et paysage dans la poésie belge francophone. Cahiers de l'Association internationale des études françaises, 63, 91-106.

Balakia, A. (1997). Histoire des poétiques. Paris: Presses universitaires de France.

Bodson-Thomas, A. (1942). L'esthétique de Georges Rodenbach. Liège: Vaillant-Carmanne.

Chenet-Faugeras, F. (1994). L'invention du paysage urbain. Romantisme, 83, 27-38.

Gorceix, P. (1992). Réalités flamandes et symbolisme fantastique. Bruges-la-Morte et Le Carillonneur. Paris: Lettres modernes. 
Maes, J. (1952). Georges Rodenbach 1855-1898. Gembloux: J. Duculot.

Marx, J. (1994). Autopsie d'un poète national. Textyles, 11, 21-34.

Mauclair, C. (1899, 15 February). Georges Rodenbach. La Revue des revues, 384-385.

Rime, J. (2016) (Ed.). Contes symbolistes: Vol. 3. Grenoble: ELLUG, Université Grenoble Alpes.

Rodenbach, G. (1884). L'Hiver mondain. Bruxelles: Henry Kistemaeckers .

Rodenbach, G. (1924). Évocations. Paris: La Renaissance du Livre.

Rodenbach, G. (1978). Euvres. Genève: Slatkine Reprints.

Rodenbach, G. (1998). Bruges-la-Morte. Paris: Flammarion.

Rodenbach, G. (2016). Le Rouet des brumes. Contes posthumes. In J. Rime (Ed.), Contes symbolistes: Vol. 3. (pp. 17-449). Grenoble: ELLUG, Université Grenoble Alpes. 